\title{
Best Approach to Prevent Diabetic Nephropathy
}

\section{Journal of Diabetes and Endocrinology Research}

Review Article

Usama A Sharaf El Din ${ }^{1 *}$, Mona M. Salem ${ }^{2}$, Dina O Abdulazim ${ }^{3}$

${ }^{I}$ Nephrology unit, Internal Medicine Department, School of Medicine, Cairo University, Egypt

${ }^{2}$ Endocrinology unit, Internal Medicine Department, School of Medicine, Cairo University, Egypt

${ }^{3}$ Rheumatology and Rehabilitation Department, School of Medicine, Cairo University, Egypt

\author{
Correspondence author \\ Professor Usama AA Sharaf EI Din \\ International Kidney Center \\ 58th Abbas El Akkad St \\ Nasr City \\ Cairo, Egypt \\ Post Code: 11759 \\ Telephone: +201111333800 \\ Fax: +20222753890 \\ E-mail: usamaaas@gmail.com
}

Submitted : 26 Oct 2019; Published : 10 Nov 2019

\begin{abstract}
The last two decades have witnessed a storm in our understanding of the pathogenesis of diabetic nephropathy. In parallel, the newly evolved hypoglycemic agents have shown different mechanisms of action that would effectively tackle the different pathogenic mechanisms involved in the development and progression of diabetic nephropathy. Several large prospective placebo controlled clinical trials have supported the significant impact of these different agents to withhold the progression of diabetic nephropathy. Almost all these trials involved type 2 diabetes mellitus (T2DM). However, most of these agents can additionally be used in typel diabetes mellitus (T1DM) patients aiming at avoidance of long-term complications of this disease. The most recent of these trial, the DECLARE - TIMI 58 renal outcome trial, have additionally declared the preventive value of dapagliflozin in T2DM. In this review, we are going to discuss the most recent and relevant pathogenic mechanisms highlight the therapeutic and value of the early use of the different agents to prevent the development of diabetic nephropathy.
\end{abstract}

Keywords: Type 1 diabetes, Type 2 diabetes, Diabetic Nephropathy, Microvascular Complications, Macrovascular Complications, DPP4Is, SGLT2Is, nrf2 agonists

\section{Introduction}

Diabetes mellitus is a pandemic disease that has exponentially increasing prevalence. In 1980, 108-million persons had diabetes worldwide while in 2014, 430- million persons were affected [1]. In spite of the increased awareness about this disease and the worldwide efforts to give optimum care, 3.7 million diabetic patients lost their lives in 2012 because of diabetes and its complication [2]. This figure exceeded 5 million deaths annually in the last few years [3]. Diabetic kidney disease (DKD) is the most common cause of end-stage renal disease (ESRD). One-third of T1DM develop ESRD, while only $10-20 \%$ of type 2 diabetes mellitus (T2DM) patients progress to ESRD [4,5]. The prevalence ofcongestive heart failure (CHF) among diabetic patients aged 55 to 64 years is 5.5 folds the prevalence among non-diabetic personnel of the same age [6]. Diabetes is an independent risk factor for the development of ischemic heart disease (IHD). CHF and IHD are the commonest causes of death in T1DM and T2DM patients [7]. Endothelial dysfunction is a common pathology underlying the etiopathogenic mechanism of all these complications [8]. This endothelial dysfunction is a sequel to many metabolic changes encountered in hyperglycemic personnel. These metabolic changes include increased oxidative stress hyperuricemia, stimulation of sodium hydrogen exchangers (NHE) and stimulation of renal sodium glucose transporters (SGLT) [9-11].

Twenty-five years ago, the Diabetes Control and Complications Trial (DCCT) research group announced 50\% reduction of microvascular complications among T1DM patients in the tight blood sugar control group compared to poorly controlled cases [12]. Five years later, the United Kingdom Prospective Diabetes Study (UKPDS) group announced similar findings among T2DM patients [13]. The last 15 years have witnessed the introduction of 
three new hypoglycemic agents, namely, glucagon-like peptide-1 receptor agonists (GLP-1RA), dipeptidyl peptidase 4 inhibitors (DPP4Is), and sodium glucose co-transporter-2 inhibitors (SGLT2Is). These three agents carry unique features, namely, the minimal incidence of hypoglycemic events and the favorable impact on body weight. GLP-1RA and SGLT2Is are associated with body weight reduction, while DPP4Is are weight neutral $[14,15]$.

Compared to older hypoglycemic agents, these newer groups carry potential favorable protective effects on endothelium, and can significantly reduce adverse cardiovascular events of diabetes in case of SGLT2Is and GLP-1RA, and are renoprotective. SGLT2Is may also prevent or withhold diabetic retinal complications [16]. This review will highlight the possible new strategy to prevent the development and progression of Diabetic nephropathy (DN), the main target of this disease management.

\section{The Endothelium in Diabetes}

The first report on the role of the endothelium as an important regulator of local vascular tone was in 1980 [17]. The vascular endothelium is an important component of diabetic complications. Endothelial dysfunction is eminent not only in diabetic patients but also in patients suffering obesity or metabolic syndrome. Decreased synthesis of nitric oxide (NO), a potent vasodilator, is the salient feature of endothelial dysfunction. Decreased NO underlies insulin resistance by reducing insulin access to target cells [18]. Insulin has also to cross endothelial cells to reach target tissues $[19,20]$. Hyperglycemia can lead to endothelial mitochondrial fragmentation and increased production of reactive oxygen species (ROS) [21]. Increased endothelial ROS is associated with increased breakdown of NO [22]. Impaired endothelial function was demonstrated within visceral fat [23], cardiac and skeletal muscles [23-24]. Endothelial dysfunction is associated with accelerated atherosclerosis in an animal model, diabetic retinopathy, nephropathy, neuropathy and cerebral dysfunction [25-29]. To affirm the role of endothelial dysfunction in the development of diabetic nephropathy, two separate studies have disclosed that endothelial nitric-oxide synthase (eNOS) deficient mice robustly develop diabetic nephropathy [30,31].

\section{Sodium Hydrogen Exchangers}

The sodium hydrogen exchangers (NHE) are trans-membrane ion channels that are responsible for intracellular $\mathrm{pH}$ regulation through the extrusion of hydrogen in exchange with sodium [Fig $1]$.

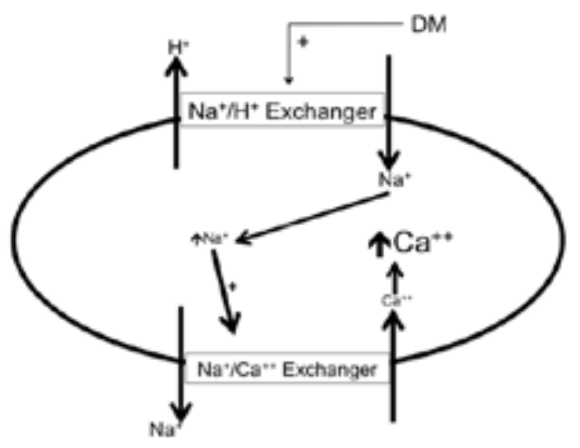

Fig. 1: Diabetic state increases the activity of the sodium/ hydrogen exchanger on the surface of endothelial cells, vascular smooth muscle cells, cardiomyocytes, and tubular epithelial cells. Consequently, intracellular and mitochondrial calcium.

NHE exist in nine isoforms $[32,33]$. NHE1 is present on the surface of endothelium, vascular smooth muscle cells (VSMCs), cardiomyocytes and platelets, while in case of renal tubular and intestinal epithelium NHE3 is encountered. Activation of the NHE1 within endothelium, VSMCs, and cardiomyocytes may underlie micro-vascular and macro-vascular complications of diabetes. It can also have a role in insulin resistance and systemic hypertension. These exchangers cause increased sodium influx that stimulates sodium-calcium exchanger with consequent increase of intracellular calcium. Within endothelium, increased cytoplasmic calcium inhibits eNOS and thus decreases NO [Fig 2].

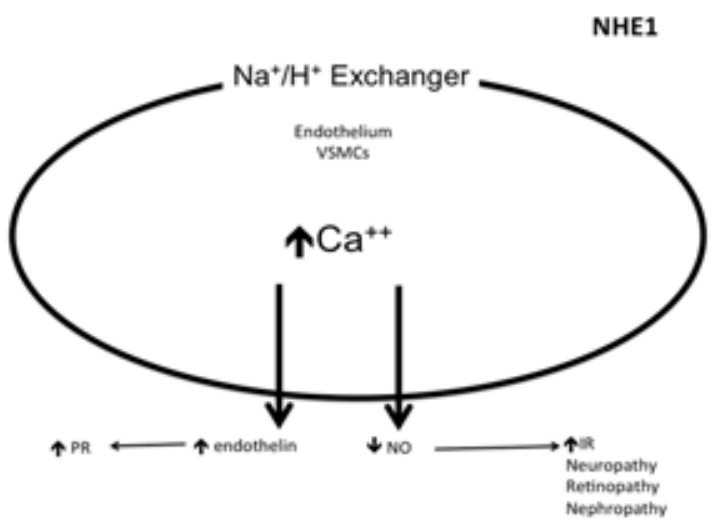

Fig. 2: increased cytosolic calcium leads to decreased nitric oxide synthesis and increased secretion of endothelin. Increased vascular smooth muscle tone leads to increased peripheral resistance and decreased tissue perfusion. This leads to decreased insulin delivery. Endothelial damage induced by calcium can also decrease permeability of the endothelium to the delivered insulin. Decrease nitric oxide production has a role in pathogenesis of neuropathy, retinopathy and nephropathy $\mathrm{PR}=$ peripheral resistance; $\mathrm{IR}=$ insulin resistance.

Increased intracellular calcium is also associated with increased intracellular and mitochondrial activity of calpain, the cysteine protease that can damage the inner mitochondrial membrane, a process that ends with cell apoptosis [34]. The development of systemic hypertension, increased insulin resistance, diabetic retinopathy, nephropathy, and neuropathy are consequences of decreased eNOS activity and accelerated endothelial senescence. It can also explain the increased frequency of vascular calcification, peripheral vascular disease, and diabetic cerebrovascular dysfunction [36]. Mitochondrial injury is associated with impaired antioxidant defense [37].

Inhibition of NHE1 using cariporide was associated with increased NO release; eNOS activity simultaneously decreased ROS production, decreased nuclear factor- $\kappa \mathrm{B}(\mathrm{NF}-\kappa \mathrm{B})$ activation and decreased the production of tumor necrosis factor- $\alpha$ and intercellular adhesion molecule-1 [38]. Increased intracellular calcium induced by NHE1 isoform on the surface of cardiomyocytes leads to cardiac hypertrophy. Peripheral 
coronary ischemia secondary to endothelial dysfunction can further activate cardiac NHE1. Active NHE1 increases intracellular and intra-mitochondrial calpain that contributes to degeneration, apoptosis, and fibrosis of myocardium [35] [Fig. 3].

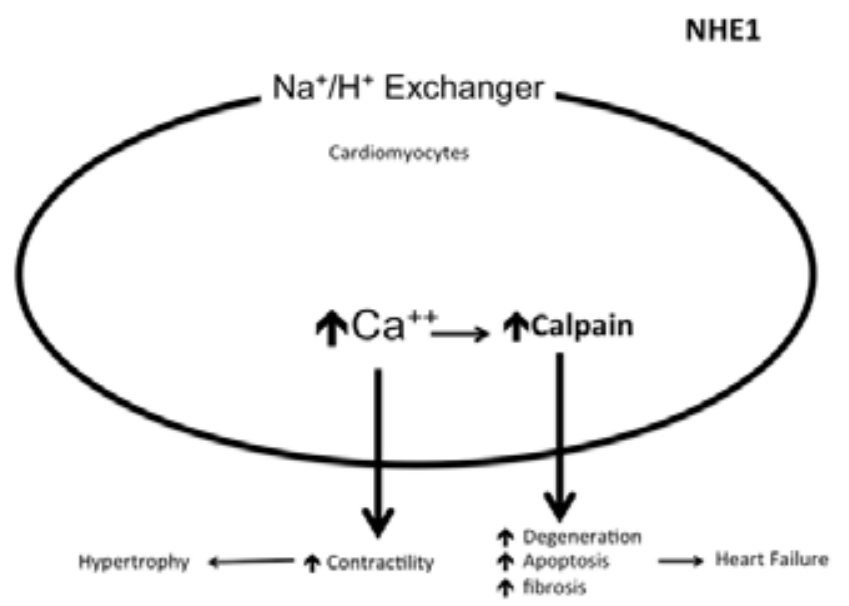

Fig. 3: increased cytosolic calcium within the cardiomyocytes leads to ventricular hypertrophy, and increased activity of the digestive enzyme calpain. This lysolethicin digests mitochondrial membranes and results in myocardial damage that finally leads to heart failure.

Activation of renal NHE3 within PCT and ascending loop of Henle causes sodium retention and can thus contribute to the development of systemic hypertension in diabetic patients $[35,39]$ [Fig. 4]

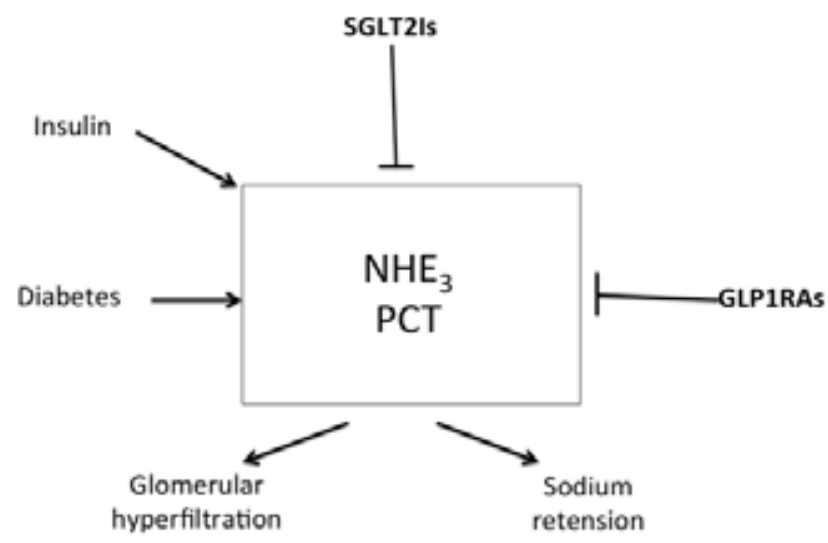

Fig. 4: increased activity of NHE3 isomer within the proximal convoluted tubules increases sodium absorption from the lumen of these tubules in exchange with the secreted hydrogen. Decreased sodium delivery to the distal nephron segments results in glomerular hyperfiltration. Diabetic state and insulin administration increase NHE3 activity while SGLT2Is and GLP1RAs inhibit it. NHE=sodium hydrogen exchanger; SGLT2Is = sodium glucose transporter- 2 inhibitors; GLP1RAs= glucagon like peptide receptor agonists.

Activation of NHE1 on the surface of platelets plays a significant role in platelet activation. This effect is mediated through increased intracellular calcium and can contribute to the pro-coagulant state in diabetes [40].
Accordingly, activation of NHE1 on the surface of endothelial cells, VSMCs, platelets, and cardiomyocytes beside the activation of renal NHE3 share in the pathogenesis of systemic hypertension, microvascular complications and macrovascular complications of diabetes that finally result in heart failure and ESRD.

\section{Oxidative Stress}

Increased oxidative stress is one of the metabolic disorders encountered in diabetes. Diabetic patients have overproduction of free oxygen radicals and decreased wash out of these radicals. Increased production of free oxygen radicals is the sequel to increased activity of nicotinamide adenine dinucleotide phosphate (NADPH) oxidase [41,42], cyclo-oxygenase [43], and lipoxygenase [44] enzymes. Hyperglycemia stimulates all these enzymes. Sodium-glucose cotransporter 2 (SGLT2) within the brush border of the proximal convoluted tubular epithelium (PCT) is another pathway of free oxygen radicals' overproduction. Increased intracellular uric acid (UA) induces NADPH oxidase [45]. Mitochondrial damage results in impaired antioxidant defense [37]. Increased free oxygen radicals activate NF- $\kappa B$ [46]. When NF- $\kappa B$ is free from its inhibitor, it translocates from the cytoplasm to the nucleus where it triggers the genes encoding transforming growth factor- $\beta 1$ (TGF- $\beta 1$ ), and monocyte chemoattractant protein-1 (MCP-1) and Intercellular Adhesion Molecule 1 (ICAM1) [47-49]. Reactive oxygen species (ROS) stimulate overproduction of protein kinase $\mathrm{C}$ (PKC) and mitogen-activated protein (MAP) kinase within mesangial cells (MCs) and pericytes. All these factors stimulate overproduction of extracellular matrix proteins [50].

\section{Uric Acid}

Serum uric acid (UA) is a strong predictor for the development of proteinuria in T1DM patients. The risk for proteinuria increases by $80 \%$ with every $1 \mathrm{mg} / \mathrm{dL}$ rise in serum UA [51]. The risk of decline of glomerular filtration rate (GFR) is significantly higher ( 2.4 folds) in T1DM patients with serum UA $>6.6 \mathrm{mg} / \mathrm{dL}$ when compared with candidates with lower level [52]. In T1DM patients followed-up for more than 18 years, serum UA was an independent predictor of overt proteinuria [53]. In T2DM patients, $68 \%$ of the hyperuricemic versus $41.5 \%$ with normal serum UA had diabetic nephropathy (DN) [54]. Further prospective studies confirmed the increased risk of development of proteinuria and accelerated decline of GFR among hyperuricemic T2DM [55,56]. Serum UA $>7 \mathrm{mg} / \mathrm{dL}$ in males and $>6 \mathrm{mg} / \mathrm{dL}$ in females were associated with a higher rate of DN progression, and overall mortality among T2DM patients that have the disease for fifteen years or more [57].

Treatment of T2DM patients suffering DN and high serum UA with allopurinol was associated with a significant decrease of urine albumin excretion (UAE) and serum creatinine and a significant increase of GFR over three years of follow-up [58]. A recent meta-analysis of 19 randomized controlled trials has confirmed the significant favorable effect of urate-lowering therapy on the rate of GFR decline. These 19 trials enrolled 992 patients [59].

Increased level of Serum UA is associated with endothelial dysfunction. High mobility group box chromosomal protein 1 (HMGB1) is a pro-inflammatory mediator synthesized and secreted by activated phagocytes or monocytes. When secreted 
extracellular, HMGB1 can interact with the receptor for advanced glycation end products (RAGE), inducing the production of multiple cytokines, and the induction of vascular adhesion molecules [60]. In a recent in vitro study, high UA concentration inhibited eNOS expression and NO production in human umbilical vein endothelial cells (HUVECs), increased extracellular HMGB1 secretion, up-regulated RAGE expression, activated NF$\kappa \mathrm{B}$, and increased the level of inflammatory cytokines. Blocking RAGE significantly suppressed the DNA binding activity of NF$\kappa \mathrm{B}$ and the levels of inflammatory cytokines [61]. High serum UA is also a significant predictor of systemic hypertension [62].

\section{Role of Glucagon-like Peptide-1 Receptor Agonists (GLP-1RA)}

Glucagon-like peptide-1 (GLP-1), is a peptide hormone secreted by the neuro-endocrine cells within the mucosa of the small intestine [63]. In healthy individuals, GLP-1 activates insulin secretion, inhibits glucagon secretion and slows gastric emptying and controls appetite [63]. The susceptibility of this peptide hormone to enzyme breakdown by the dipeptidyl peptidase-4 enzyme (DPP-4) is responsible for the very short plasma half-life of GLP-1. It cannot be used therapeutically except as continuous intravenous infusion [64]. GLP-1RA are exogenous GLP-1 analogues with variable sequence similarity to the human GLP-1 [65]. The variability involved mainly two sites in the GLP-1 molecule susceptible to cleavage by DPP4; namely, alanine and lysine at positions 8 and 34 respectively. These changes, beside other modifications, have helped to find out many peptides that simulate GLP-1 action but with longer half-life [64]. GLP-1RAs were found to decrease body weight, and some cardiovascular morbidity, without increasing the risk of hypoglycemia [66]. Robust indications for GLP-1RAs in T2DM patients not responding to metformin monotherapy, dual therapy, or insulin include overweight, inability to control appetite, high risk of cardiovascular disease, and the need of high doses of insulin [64]. The use of GLP-1 RAs can also lower systolic, and to a minor degree, diastolic blood pressure [67].

However, long term use of GLP-1 RAs was frequently reported to be associated with increased heart rate $[67,68]$. The current evidence does not support any beneficial effect of GLP-1RAs in patients with heart failure and/or impaired ventricular function $[69,70]$. The Evaluation of Lixisenatide in Acute Coronary Syndrome (ELIXA) trial was the first cardiovascular outcome trial (CVOT) of GLP-1RAs in T2DM. Based on this trial, treatment with lixisenatide in addition to conventional therapy had no impact on the cardiovascular risk in patients with T2DM and recent acute coronary syndrome [71]. In the Liraglutide Effect and Action in Diabetes: Evaluation of Cardiovascular Outcome Results (LEADER) trial, that appeared in 2016, liraglutide use significantly decreased mortality from any cause and cardiovascular events in patients with T2DM at high risk for cardiovascular events. The benefit of liraglutide treatment is more prounced in patients with eGFR $<60 \mathrm{~mL} / \mathrm{min} / 1.73 \mathrm{~m} 2$ and patients aged 50 years or more. In spite of these benefits, hospitalization rate for heart failure was not different between liraglutide and placebo groups [72]. Although the incidence of retinopathy was similar in this trial, the chance of development of nephropathy was significantly lower in patients treated with liraglutide [72]. In SUSTAIN-6 trial, semaglutide was associated with a significant decrease in the incidence and progression of nephropathy. However, a higher percentage of patients in semaglutide group developed retinopathy. Semaglutide was also associated with a $26 \%$ reduction in the hazard of cardiovascular mortality, non-fatal myocardial infarction, or nonfatal stroke. In addition, the rate of new or worsening nephropathy were lower in the semaglutide group [73].

In EXSCEL trial, extended release exenatide failed to show favorable cardiovascular impact [74]. This result could be due to the broader T2DM population studied in EXSCEL trial as regard to age and cardiovascular risk, the shorter follow-up period, the lower $\mathrm{HbA} 1 \mathrm{c}$ levels and the concomitant hypoglycemic treatment (SGLT2Is were frequently used in the placebo group) [75]. A meta-analysis including nine randomized trials with dulaglutide in 6010 T2DM patients has shown that $0.67 \%$ of patients treated with dulaglutide vs. $1.18 \%$ of the placebo group developed one of the endpoints. This difference was not significant [76].

Other glucose-independent effects of GLP-1RAs include favorable effects on blood pressure, dyslipidemia, inflammation, and fibrosis. Through inhibition of renal NHE3, GLP-1RAs can promote natriuresis and diuresis. Additional effects include inhibition of the intrarenal renin-angiotensin system, inflammation, and apoptosis. The mechanism of these effects is still not recognised. Recent studies suggest important antioxidant and anti-apoptotic activities of GLP-1RAs [77] [Fig.5].

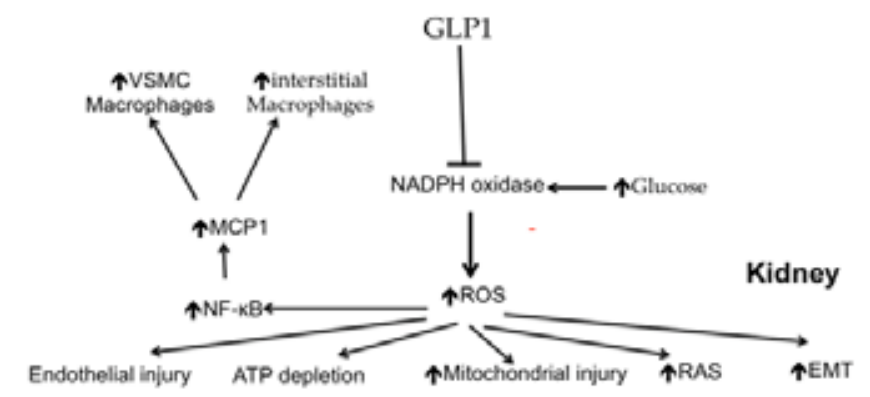

Fig. 5: Hyperglycemia stimulates NADPH oxidase enzyme within different organs including the kidney. Consequent increased production of free oxygen radicals results in increased cascade of degenerative and inflammatory processes that underlie pathology of the diabetic kidney. Glucagon like peptides inhibit NADPH oxidase and thus can muffle development or progression of diabetic nephropathy. GLP1= Glucagon like peptides; $\mathrm{NADPH}=$ nicotinamide adenine phosphate; $\mathrm{ROS}=$ reactive oxygen species; NF-кB- nuclear factor kappa B; $\mathrm{MCP} 1=$ macrophage chemoattractant fpeptide; $\mathrm{VSMCs}=$ vascular smooth muscle cells; ATP=adenosine triphosphate; $\mathrm{RAS}=$ reninangiotensin system;EMT $=$ epithelial mesenchymal transition.

A major drawback on GLP1RA is the need of frequent injections. Recently oral Semaglutide proved to be as efficacious to parenteral formula in glycemic and body weight control [78]. However, the cardiovascular and renal effects of this oral formula is not yet established. 


\section{Dipeptidyl Peptidlase 4 Inhibitors}

The discovery of non-enzymatic functions for DPP4 within the kidney has attracted the attention for the reno-protective action of this hypoglycemic agent especially after disclosure of the antiproteinuric effect of saxagliptin in T2DM patients during "Saxagliptin Assessment of Vascular Outcomes Recorded in Patients with Diabetes Mellitus-Thrombolysis in Myocardial Infarction 53" (SAVOR-TIMI 53) trial [79-83]. Experimental pharmacologic and genetic inhibition of DPP4 had also proven efficacy in preventing progressive renal damage in animal models of acute and chronic kidney disease $[84,85]$.

The glucose-lowering action of DPP4Is is through inhibition of breakdown of endogenous GLP and glucose-dependent insulinotropic peptide (GIP). These incretins improve the sensitivity of pancreatic $\beta$ cells to glucose [86]. DPP4 exists in 2 forms; membrane-bound and soluble forms [87]. Membrane-bound DPP4 is present on the cell membrane of epithelial cells in the kidneys, lungs, and small intestine.

It also exists on endothelial, and immune cells [88-90]. DPP4 on the surface of immune cells is also known as cluster of differentiation 26 (CD26) [89,90]. The soluble form (sDPP4) is the consequence of shedding of the membrane-bound form. sDPP4 level increases in obese subjects and T2DM patients and may participate in increased insulin resistance in these cases [91]. Membrane-bound DPP4 expression is triggered in case of hypoxia as well as its' shedding [92,93].

Within the kidney, DPP4 in S1-S3 segments of the proximal convoluted tubules (PCT) are linked to NHE3 and plays a role in salt and water retention through stimulation of this exchanger, NHE3 activity decreases on inhibition of angiotensin II synthesis by captopril [94], or inhibition of DPP4 [95]. Angiotensin II inhibits megalin receptor endocytosis protein expression. This process is reversed by DPP4Is [96]. Treatment with DPP4 inhibitors may reverse reduced uptake of albumin and other low molecular weight proteins by PCT [97]. DPP4 was also discovered on the glomerular endothelium and the base of the foot processes of podocytes [98]. DPP4 is expressed on T-cells, B-cells, macrophages, and dendritic cells in the kidney [90]. Stimulation of DPP4 on the surface of different immune and inflammatory cells may mediate inflammation within the kidney in diabetic patients. DPP4Is decrease inflammation in diabetes. This finding suggests inflammation as an eminent player in DPP4-mediated kidney injury [99].

However, in spite of the reduction in urine albumin excretion observed in 3 randomized controlled studies (RCT) in T2DM patients treated with DPP4Is [100-102], the only study that specifically looked for the anti-proteinuric effect of linagliptin failed to find a significant impact [103]. Moreover, DPP4Is failed to have a significant impact on doubling of serum creatinine, change in GFR, or ESRD [100-102]. On the other hand, administration of linagliptin to T2DM patients that had renal dysfunction and were prescribed ACE inhibitors or ARBs has led to additive significant reduction in albuminuria [104].

In normoglycemic milieu, microRNA-29 (miR29) suppresses DPP4 gene. In hyperglycemic state, this suppression is lost. As a consequence, cell surface DPP4 activity increases [105]. In diabetic mice, activated endothelial DPP4 induces phosphorylation of adjacent integrin $\beta 1$ on the surface of the endothelium. The activated DPP4, together with the phosphorylated integrin $\beta 1$ form a complex that up-regulates TGF $\beta$ receptor and activates the surface vascular endothelial growth factor receptor type 1(VEGFR1). Upregulated TGF $\beta$ receptor and VEGFR1 stimulate endothelialmesenchymal transition (EndMT) that increases transition to fibroblasts with subsequent increased fibrogenesis [106] [Fig.6].

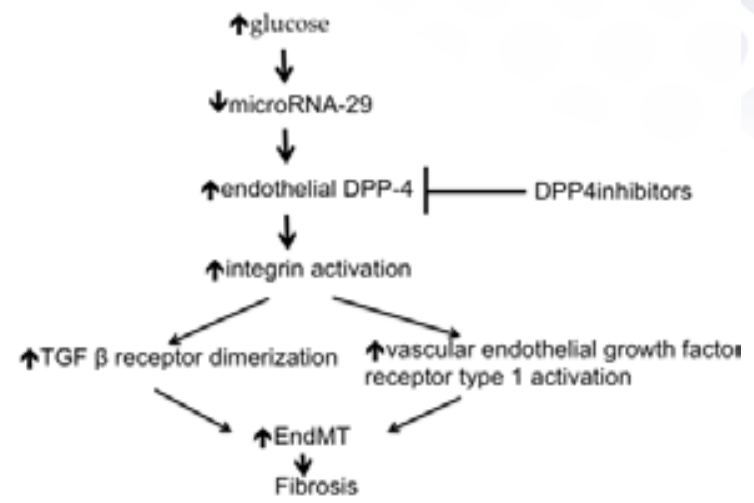

Fig. 6: MicroRNA-29 is a natural inhibitor of endothelial DPP-4 within renal vasculature. Hyperglycemia inhibits microRNA-29 and thus stimulates endothelial DPP-4. DPP-4= dipeptidyl peptidase; $\mathrm{TGF}=$ transforming growth factor; EndMT $=$ endothelial mesenchymal transition.

However, the lack of significant impact ofDPP4Is on GFR in human studies would cast doubts on their favorable effect on renal fibrosis in humans. The effect ofDPP4Is treatment on the retina is debatable. While some investigators reported an increase in retinal endothelial leakage and vascularity [107], others have reported a significant reduction in the risk of diabetic retinopathy progression [108].

The lack of the expected favorable effect of DDP4Is on diabetic microvascular and macrovascular complications of diabetes in spite of the proven molecular and experimental mechanisms can be attributed to potentiation of the stem cell chemokine, stromal cell-derived factor-1 (SDF-1), which promotes inflammation, proliferation and neovascularization [109]. SDF-1 enhances atheromatous plaque growth and instability, cardiac inflammation, and fibrosis [110]. The renal effects of DPP4Is are mainly through potentiation of SDF-1 leading to podocyte injury and glomerulosclerosis. SDF-1 also induces natriuresis in the distal tubules, contrary to SGLT2Is and NHE3 inhibitors that act on PCT. Hence, SDF-1 cannot utilize tubuloglomerular feedback to modulate the glomerular hyperfiltration [109,111] [Fig.7]. 


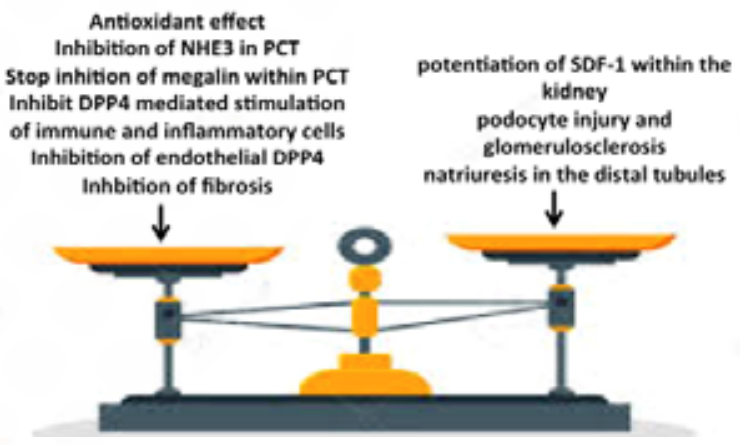

Fig.v 7: The beneficial effects of DPP4Is on the kidney are muffled by the bad effect induced by stromal cells derived factor 1(SDF-1). DPP-4= dipeptidyl peptidase; TGF $=$ transforming growth factor; EndMT= endothelial mesenchymal transition.

\section{Sodium-Glucose Co-transporters Inhibitors}

SGLT2Is constitute a recently introduced group that has insulin independent hypoglycemic effect. Three members of this group, namely empagliflozin, canagliflozin, and dapagliflozin are FDA approved and are now used worldwide. By inhibiting the upregulated SGLT2 co-transporters in the brush border of the S1 segment of the PCT, SGLT2Is can reduce the renal threshold for plasma glucose from 196 to $22 \mathrm{mg} / \mathrm{dL}$, thereby enhancing urinary excretion of glucose[112]. Theyalso increasedistal sodiumdelivery and hence distal tubular sodium absorption. Increased adenosine triphosphate (ATP) consumption during sodium absorption with a consequent increase of adenosine production, causes afferent arteriolar vasoconstriction and fall in renal blood flow, reverses hyperfiltration, and accordingly reduces renal injury [Fig. 8]
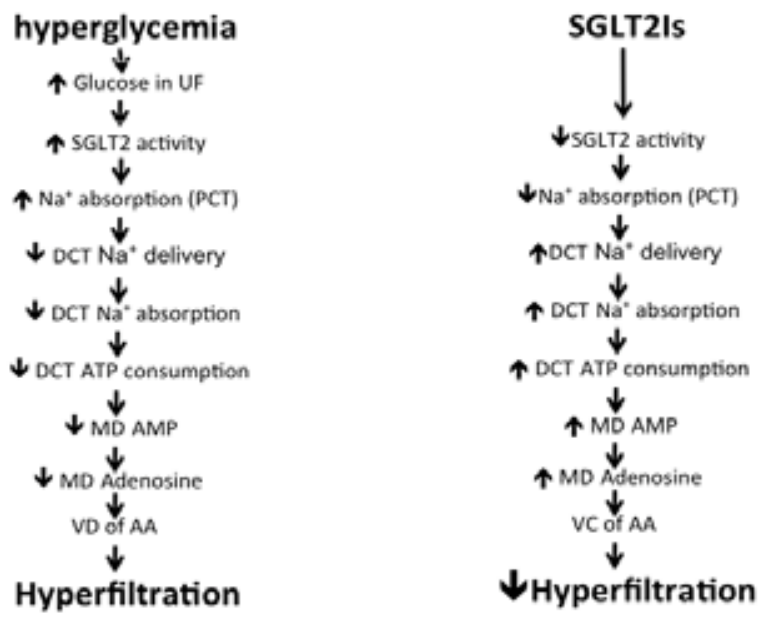

Fig. 8: Mechanism of hyperfitration induced by hyperglycemia and howdoSGLT2Iscontrolit. UF=ultrafiltrate;SGLT=sodiumglucose transporter; $\mathrm{Na}+=$ sodium; $\mathrm{PCT}=$ proximal convoluted tubules; $\mathrm{DCT}=$ distal convoluted tubules; $\mathrm{ATP}=$ adenosine triphosphate; $\mathrm{MD}=$ macula densa; $\mathrm{AMP}=$ adenosine monophosphate; $\mathrm{VD}=$ vasodilatation; $\mathrm{AA}=$ afferent arteriole $; \mathrm{VC}=$ vaso-constriction .

In addition, SGLT2Is exert other beneficial effects, including reductions in body weight, serum UA, and blood pressure [113]. Excess glucose within the tubular lumen triggers the uric acid transporter GLUT9 within the S3 segment of the PCT and in the collecting duct to excrete UA in exchange with glucose [114]. The antihypertensive effect of SGLT2Is is related to volume depletion, loss of body weight, inhibition of endothelial NHE1 and renal NHE3, and reduction in serum UA [62].

SGLT2Is not only decrease serum UA but they can decrease intracellular fructose metabolism and UA synthesis in the PCT epithelium [115]. Intracellular UA is pro-oxidant. It stimulates NADPH oxidase enzyme activity with consequent increase in production of ROS.

This leads to premature senescence of these cells, activation of the renin-angiotensin system, epithelial-mesenchymal transition, and activation of the inflammatory cascade through activation of NF-kB [116-118] [Fig. 5]. Cyclin-dependent kinase (CDK) inhibits cell senescence. P21 is an inhibitor of CDK and thus promote cell senescence. Hyperglycemia induces P21 while SGLT2Is inhibit this factor within PCT cells [119,120] [Fig.9].

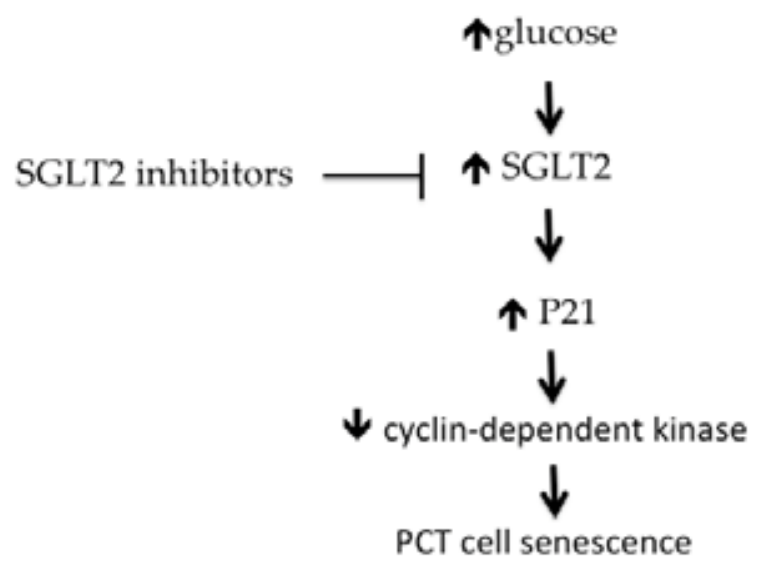

Fig. 9: activation of SGLT2 in diabetic patients leads to overactivity of P21, the natural inhibitor of Cyklin-dependent kinase 2. This kinase enzyme inhibits cell senescence. By inducing P21, diabetic patients suffer increased proximal tubular epithelium senescence. Through inhibition of SGLT2, SGLT2Is ptotect proximal tubular epithelial cells against increased senescence. SGLT=sodium glucose transporter; $\mathrm{PCT}=$ proximal convoluted tubule.

SGLT2Is also dampen the expression of Toll-like receptor-4, the binding of nuclear DNA for activator protein 1, the increased collagen IV expression as well as the increase in interleukin-6 secretion and interstitial macrophage infiltration induced by hyperglycemia within the renal parenchyma [121]. Moreover, fibrotic and inflammatory genes are suppressed within the diabetic kidney by SGLT2Is $[122,123]$.

Through suppression of intracellular UA production, SGLT2Is inhibits renal gluconeogenesis. Intracellular UA stimulates adenosine monophosphate dehydrogenase (AMPD) enzyme and inhibits adenosine monophosphate kinase (AMPK) enzyme activities. Intracellular AMPD stimulates while AMPK inhibits gluconeogenesis [124]. In healthy personnel, the kidneys participate in endogenous glucose production. In the fasting state, $20 \%-25 \%$ of endogenous glucose production takes place through renal gluconeogenesis. In T2DM, renal gluconeogenesis increases threefold [125]. 
Empagliflozin in EMPA-REG trial achieved 55\% reduction of the chance of ESRD in T2DM patients with established cardiovascular disease, and an eGFR $>30 \mathrm{~mL} / \mathrm{min} / 1.73 \mathrm{~m} 2$.

The median observation time in EMPA-REG trial was 3.1 years [126]. In comparison, losartan treatment of similar population having DN has led to a $28 \%$ delay in the onset of ESRD during a mean follow-up of 3.4 years [127]. Empagliflozin treatment resulted in a $39 \%$ reduction of incident or worsening nephropathy, a $38 \%$ reduction in progression to overt albuminuria and a $44 \%$ reduction in doubling of serum creatinine [128]. The favorable outcome of SGLT2Is is attributable to their effect on glomerular hyperfiltration, blood pressure, body weight, and serum UA in diabetic patients [128-130]. SGLT2Is also inhibit NHEs on the surface of cardiomyocytes, endothelial cells, and renal tubular epithelial cells. NHE inhibition can explain the distinguished cardioprotective and renoprotective actions of SGLT2Is [131133]. Decreased renal blood flow induced by SGLT2Is is related to tubuloglomerular feedback and not related to the renin-angiotensin system (RAS) blockade. Empagliflozin and dapagliflozin increase plasma aldosterone and angiotensin II $[134,135]$, together with increased activity of urinary angiotensin converting enzyme and angiotensin converting enzyme2 [136].

2-years treatment of T2DM patients (total of 1450 cases) already kept on metformin with either once-daily canagliflozin $100 \mathrm{mg}$, canagliflozin $300 \mathrm{mg}$, or glimepiride titrated to $6-8 \mathrm{mg}$ resulted in eGFR decline by $0.5,0.9$, and $3.3 \mathrm{~mL} / \mathrm{min} / 1.73 \mathrm{~m} 2 /$ year respectively $(\mathrm{P}<0.01$ for each canagliflozin group versus glimepiride) in spite of comparable reductions in $\mathrm{HbAlc}$. UAE declined more with canagliflozin $100 \mathrm{mg}$ or canagliflozin 300 $\mathrm{mg}$ than with glimepiride. These results further support that the renoprotective effect of SGLT2Is is independent of their glycemic effect [137]. Contrary to DPP4Is and sulfonylureas that are significantly associated with increased risk of diabetic retinopathy, SGLT2Is were not associated with a higher risk of diabetic retinopathy than placebo among 100928 patients with T2DM included in 37 independent randomized controlled trials with 1806 diabetic retinopathy events [138]. In the Canagliflozin Cardiovascular Assessment Study (CANVAS), 10142 T2DM patients were assigned to canagliflozin or placebo. $34 \%$ of the patients had $\geq 2$ risk factors for cardiovascular events but had no history of previous cardiovascular event (primary prevention cohort), while the remaining $66 \%$ had a positive history of cardiovascular event (secondary prevention cohort). The patients were randomly assigned in a ratio of $1: 1: 1$ to either canagliflozin $100 \mathrm{mg}$, canagliflozin $300 \mathrm{mg}$ or matching placebo. After treatment for a mean of 3.6 years, the primary endpoint (cardiovascular death, nonfatal myocardial infarction, or nonfatal stroke) has occurred less frequently with canagliflozin compared with placebo (26.9 versus $31.5 / 1000$ patient-years; $\mathrm{P}=0.02$ ). There was no statistical evidence of heterogeneity between the primary and secondary prevention cohorts.

Renal outcomes were reduced by $40 \%$ and heart failure hospitalization was reduced by $33 \%$ in patients treated with canagliflozin $[139,140]$. In the DECLARE -TIMI 58 trial, 17,160 T2DM patients, including 6,974 with atherosclerotic cardiovascular disease, were assigned for $10 \mathrm{mg}$ Dapagliflozin or placebo in 1:1 ratio and were followed for a median of 4.2 years. Dapagliflozin decreased the composite of cardiovascular death or hospitalizations for heart failure in those with established atherosclerotic cardiovascular disease (ASCVD) and those with only multiple risk factors [141]. When patients with previous myocardial infarction $(n=3,584)$ were specifically looked for, adverse cardiovascular events were $16 \%$ less in the dapagliflozin arm [142]. According to these studies, SGLT2Is should be prescribed aiming at cardiovascular protection in patients with T2DM and ASCVD [143]. In addition, the renal outcome results of the DECLARE - TIMI 58 have supported the favorable renoprotective effects of SGLT2Is. $47.6 \%$ of the patients in this trial had GFR $>90,45.1 \%$ had GFR between 60 and 90 , while only $7.4 \%$ of the patients had GFR $<60 \mathrm{~mL} / \mathrm{min} / 1.73 \mathrm{~m} 2$. More than twothirds of the recruited patients had normal urine albumin excretion.

In CREDENCE trial, T2DM patients suffering CKD and albuminuria (4400 patients) were randomly assigned to receive canagliflozin $100 \mathrm{mg}$ daily or placebo in $1: 1$ ratio. All the patients had an eGFR of 30 to $<90 \mathrm{~mL} /$ minute $/ 1.73 \mathrm{~m} 2$ and albuminuria (urine albumin/creatinine ratio $>300$ to $5000 \mathrm{mg} / \mathrm{gm}$ ) that were receiving RAS blockers. The primary outcome was a composite of ESRD (dialysis, transplantation, or a sustained eGFR of $<15 \mathrm{~mL} /$ $\mathrm{min} / 1.73 \mathrm{~m} 2$ ), a doubling of the serum creatinine, or death from renal or cardio-vascular causes. The projected duration of the study was 5.5 years. Investigators of this study prematurely terminated the trial after a planned interim analysis on the recommendation of the data and safety monitoring committee. This analysis has shown a highly significant reduction of the primary composite endpoint by $34 \%$ in patients treated with canagliflozin after 2.6 years of treatment. Patients in the canagliflozin group also had a lower risk of ESRD, hospitalization for HF, and the composite of $\mathrm{CV}$ death, myocardial infarction, or stroke. These results indicate that canagliflozin is an effective treatment for renal and cardiovascular protection in T2DM patients suffering CKD [144]. The observed benefits were obtained mainly in patients whose basal eGFR was between 30 and $<60 \mathrm{~mL} / \mathrm{min} / 1.73 \mathrm{~m} 2$. The hypoglycemic effect of SGLT2Is is almost lost when eGFR is lower than $45 \mathrm{~mL} / \mathrm{min} / 1.73 \mathrm{~m} 2$. In addition, these findings were observed despite very modest differences in blood sugar, weight, and blood pressure between the placebo and the active treatment groups. This suggests that the mechanism of benefit is independent of glycemic control and is likely related to the reduction in single nephron hyperfiltration related to NHE3 inhibition.

Contrary to CREDENCE trial patients where all patients were prescribed RAS blockers, only $81.3 \%$ of DECLARE study patients were on RAS blockers. The prespecified composite cardio-renal endpoints ( $\geq 40 \%$ decrease in estimated glomerular filtration rate to $<60 \mathrm{~mL}$ per minute per $1.73 \mathrm{~m} 2$ of body-surface area, new end-stage renal disease, or death from renal or cardiovascular causes) were significantly reduced by $24 \%$ in the dapagliflozin group while the prespecified composite renal endpoints decreased by $47 \%$, and the chance to develop ESRD decreased by $56 \%$ in the dapagliflozin group. The significant impact of dapagliflozin was encountered in patients having baseline GFR $>90$, between 60 and 90, in normo-albuminuric patients, in patients with microalbuminuria and in those with overt proteinuria. These favorable effects were only encountered in patients already maintained on either ACE inhibitors or ARBs [145]. 
in single nephron hyperfiltration related to NHE3 inhibition.

Contrary to CREDENCE trial patients where all patients were prescribed RAS blockers, only $81.3 \%$ of DECLARE study patients were on RAS blockers. The prespecified composite cardio-renal endpoints $(\geq 40 \%$ decrease in estimated glomerular filtration rate to $<60 \mathrm{~mL}$ per minute per $1.73 \mathrm{~m} 2$ of body-surface area, new end-stage renal disease, or death from renal or cardiovascular causes) were significantly reduced by $24 \%$ in the dapagliflozin group while the prespecified composite renal endpoints decreased by $47 \%$, and the chance to develop ESRD decreased by $56 \%$ in the dapagliflozin group. The significant impact of dapagliflozin was encountered in patients having baseline GFR $>90$, between 60 and 90, in normo-albuminuric patients, in patients with microalbuminuria and in those with overt proteinuria. These favorable effects were only encountered in patients already maintained on either ACE inhibitors or ARBs [145].

\section{Free oxygen radicalls scavengers}

Many preclinical studies have overwhelmed the role of reactive oxygen species (ROS) in the pathogenesis of diabetic complications. However, the less favorable outcomes of different antioxidants to prohibit the development or progression of diabetic complications in large clinical trials have dampened the enthusiasm for the use of antioxidant agents in diabetes [146]. Clinical studies using vitamin A, C, and E as antioxidant agents in pre-diabetic and T2DM patients were disappointing. Nuclear factor erythroid 2-related factor 2 (Nrf2) is a transcription factor that protects and restores cell homeostasis upon activation. Although Nrf2 is activated in response to hyperglycemia, this activation does not reach the sufficient level capable to combat the oxidative stress fueled by hyperglycemia [147].

\section{Recommendations of Diabetes Associations}

InOctober 2018, the European Association for the Study ofDiabetes (EASD) and the American Diabetes Association (ADA) issued an updated consensus statement on management of hyperglycemia in T2DM patients. This consensus was released during the annual meeting of EASD in Berlin, Germany. In this consensus, patients with clinical CV disease should receive one of SGLT2Is or GLP1RAs, while in patients with CKD or clinical HF and ASCVD, SGLT2Is should be considered [148]. The choice of diabetes therapies as recommended by the American Association of Clinical Endocrinologists (AACE) and American College of Endocrinology (ACE) must be individualized based on many attributes including the risk reduction in heart and kidney disease [149].

\section{Novel Markers of Diabetic Complications}

Mannose-binding lectin (MBL) is a recognized protein of the innate immune system. It is composed of a lectin (carbohydrate-binding) moiety attached to a collagenous moiety.

MBL binds to a wide range of sugars that permits MBL to interact with a wide range of viruses, bacteria, yeasts, fungi, and protozoa containing such sugars within their cell walls or membranes. When bound to its target sugar moiety, MBL can activate the complement system in the classic pathway or in C1-independent manner [150]. MBL is independently associated with HbA1c among diabetic patients [151]. MBL is involved in complement activation within the diabetic kidney [152] and was discovered as a possible independent predictor of $\mathrm{DR}, \mathrm{DN}$ and other vascular complications in type 1 and type 2 diabetes [153-158].

In 297 newly diagnosed T2DM patients, serum fibrinogen was a strong predictor for DN [159]. Serum Adiponectin was proved as a strong predictor of DN in both type 1 and type 2 diabetic patients according to a recent meta-analysis of 13 studies of more than five thousand cases [160].

\section{Discussion}

Diabetic nephropathy poses a massive public health and economic burden. The introduction of GLP1RAs, DPP4Is, and SGLT2Is has revived the hope to effectively prevent or slow down the rate of progression of this complication. These hypoglycemic agents have, in addition, a favorable effect on body weight with less likelihood to experience hypoglycemia. In parallel with their evolving evidence of $\mathrm{CV}$ and renal protective effects, ADA recommended SGLT2Is as second- or third-line antihyperglycemic treatment [161]. The updated consensus statement on management of hyperglycemia in type 2 diabetes issued by EASD and ADA has also recommended the early introduction of SGLT2Is and GLP1RAs to diabetic patients with clinical CV disease and SGLT2Is to patients with CKD or clinical HF and ASCVD. These recommendations were founded on the accumulating evidence of the significant impact of these agents in secondary prevention. The lack of similar significant impact in primary prevention is likely related to the relatively short duration of CVOTs. The most famous primary prevention trial in T2DM patients is UKPDS. It took ten years after the end of this study to get significant differences in acute myocardial infarction and overall mortality between intensive therapy group and the standard of care group [162]. In spite of the significant renoprotective effect of canagliflozin in CREDENCE trial in the whole studied group, patients with $\mathrm{eGFR} \geq 60 \mathrm{~mL} / \mathrm{min} / 1.73 \mathrm{~m} 2$ and patients with UAE $\leq 1000 \mathrm{mg} / \mathrm{gm}$ creatinine failed to get the expected benefit [144]. The planned duration of this study was 5.5 years. However, the study was prematurely terminated according to the observed significant difference in the composite endpoints between the 2 arms in the whole group. Given the known long duration of stage $4 \mathrm{DN}$, the short duration of this study was not enough for patients recruited with $\mathrm{eGFR} \geq 60 \mathrm{~mL} / \mathrm{min} / 1.73 \mathrm{~m} 2$ and patients with $\mathrm{UAE} \leq 1000 \mathrm{mg} / \mathrm{gm}$ creatinine to develop enough number of primary endpoints. The rate of decline of GFR in DN patients is around $6 \mathrm{~mL} / \mathrm{min} / 1.73 \mathrm{~m} 2$ [137]. The more recent results of the DECLARE -TIMI 58 did support this view. This last mentioned trial continued for 4.2 years. In this study, the significant impact of dapagliflozin was encountered in patients having baseline GFR $>90$, between 60 and 90, in normoalbuminuric patients, and in patients with microalbuminuria. These favorable effects in patients of DECLARE study are likely related to the relatively longer duration of follow-up [145, 163]. Taking these results into account and according to the accumulating evidence, more energetic primary preventive studies should be designed. These new studies should select newly diagnosed diabetic patients that have laboratory markers of likelihood to develop diabetic nephropathy. The main obstacle for such studies is the duration needed to have enough number of endpoints for adequate statistical analysis. Such long duration may lend such studies very J Diabetes Endocrinol Res; $2019 \quad$ www.unisciencepub.com Volume $1 \mid$ Issue 1 
costly and very exhaustive. Given the safety and non-inferiority of SGLT2Is, GLP1RAs, and DPP4Is, and the highlighted beneficial effects of these agents, we suggest a more reproducible approach to manage T2DM patients. In addition to T2DM patients with clinical CV disease, and those with ASCVD, patients with high cardiovascular risk should be prescribed SGLT2Is as second-line hypoglycemic agent after metformin. RAS blockers should be additionally added to guarantee optimum benefit. In the case of morbid obesity, inability to control food avidity or hyperglycemia, GLP1RAs can be used instead of SGLT2Is. In case of failure of SGLT2Is to achieve the glycemic target, either DPP4I or GLP1RA should be added as the third-line agent. SGLT2I can be added on top of GLP1RA if the later failed to achieve the glycemic target. Routine screening of diabetic patients for likelihood to develop diabetic nephropathy using the early predictors like serum MBL, fibrinogen, or adiponectin can help to select patients prone to develop diabetic nephropathy. These patients should be prescribed SGLT2Is to prevent the development of the disease instead of waiting until signs of renal involvement develop. This primary prevention approach can completely abort the development of DN instead of the current secondary prevention that just postpones the event for few months or years.

\section{Conflict of Interest}

The authors have nothing to declare

\section{References}

1. Hu FB, Satija A, Manson JE (2015) Curbing the Diabetes Pandemic: The Need for Global Policy Solutions. JAMA 313: 2319-2320.

2. Diabetes Mellitus - epidemiology (2016) World health organization Global report on diabetes.

3. Ingelfinger JR, Rosen CJ (2019) Clinical Credence - SGLT2 Inhibitors, Diabetes, and Chronic Kidney Disease. N Engl J Med 380: 2371-2373.

4. Humphry LL, Ballard DJ, Frohnert PP, Chu CP, O'Fallon WM, et al. (1989) Chronic renal failure in non-insulin dependent diabetes mellitus. A population-based study in Rochester, Minnesota. Ann Intern Med 111: 788-796.

5. DeFronzo RA (1995) Diabetic nephropathy: etiologic and therapeutic considerations. Diabetes Rev 3: 510-564.

6. Nichols GA, Hillier TA, Erbey JR, Brown JB (2001) Congestive heart failure in type 2 diabetes: prevalence, incidence, and risk factors. Diabetes Care 24: 1614-1619.

7. Zeadin MG, Petlura CI, Werstuck GH (2013) Molecular mechanisms linking diabetes to the accelerated development of atherosclerosis. Can J Diabetes 37: 345-350.

8. Packer M (2017) Activation and Inhibition of SodiumHydrogen Exchanger Is a Mechanism That Links the Pathophysiology and Treatment of Diabetes Mellitus With That of Heart Failure. Circulation 136: 1548-1559.

9. Maiese K (2015) New Insights for Oxidative Stress and Diabetes Mellitus. Oxid Med Cell Longev 2015: 875961.
10. Cai W, Duan XM, Liu Y, Yu J, Tang YL, et al. (2017) Uric Acid Induces Endothelial Dysfunction by Activating the HMGB1/RAGE Signaling Pathway. Biomed Res Int 2017: 4391920.

11. List JF, Whaley JM(2011) Glucose dynamics and mechanistic implications of SGLT2 inhibitors in animals and humans. Kidney Int Suppl 120: S20-27.

12. Cleary P, Crofford O, Davis M, Rand L, Siebert C, et al. (1993) The effect of intensive treatment of diabetes on the development and progression of long-term complications in insulin-dependent diabetes mellitus. N Engl J Med 329: $977-$ 986.

13. UK Prospective Diabetes Study (UKPDS) Group (1998) Intensive blood-glucose control with sulphonylureas or insulin compared with conventional treatment and risk complications in patients with type 2 diabetes (UKPDS 33). Lancet 352: 837-853.

14. Røder ME (2018) Major adverse cardiovascular event reduction with GLP-1 and SGLT2 agents: evidence and clinical potential. Ther Adv Chronic Dis 9: 33-50.

15. Triplitt C, Solis-Herrera C, Cersosimo E, Abdul-Ghani M, Defronzo RA (2015) Empagliflozin and linagliptin combination therapy for treatment of patients with type 2 diabetes mellitus. Expert Opin Pharmacother 16: 2819-2833.

16. Wakisaka M, Nagao T (2017) Sodium glucose cotransporter 2 in mesangial cells and retinal pericytes and its implications for diabetic nephropathy and retinopathy. Glycobiology 27 : 691-695.

17. Furchgott RF, Zawadzki JV (1980) The obligatory role of endothelial cells in the relaxation of arterial smooth muscle by acetylcholine. Nature 288: 373-376.

18. Roy D, Perreault M, Marette A (1998) Insulin stimulation of glucose uptake in skeletal muscles and adipose tissues in vivo is NO dependent. Am J Physiol 274: E692-E699.

19. Wang H, Wang AX, Barrett EJ (2011) Caveolin-1 is required for vascular endothelial insulin uptake. Am J Physiol Endocrinol Metab 300: E134-E144.

20. Kolka CM, Bergman RN (2013) The endothelium in diabetes: its role in insulin access and diabetic complications. Rev Endocr Metab Disord 14: 13-19.

21. Shenouda SM, Widlansky ME, Chen K, Xu G, Holbrook M, et al. (2011) Altered mitochondrial dynamics contributes to endothelial dysfunction in diabetes mellitus. Circulation 124: 444-453.

22. Sharma A, Bernatchez PN, de Haan JB (2012) Targeting endothelial dysfunction in vascular complications associated with diabetes. Int J Vasc Med 2012: 750126.

23. Farb MG, Ganley-Leal L, Mott M, Liang Y, Ercan B, et al. (2011) Arteriolar Function in Visceral Adipose Tissue Is Impaired in Human Obesity. Arterioscler Thromb Vasc Biol 32: 467-473. 
24. Liu J, Jahn LA, Fowler DE, Barrett EJ, Cao W, et al. (2011) Free fatty acids induce insulin resistance in both cardiac and skeletal muscle microvasculature in humans. J Clin Endocrinol Metab 96: 438-446.

25. Rask-Madsen C, Li Q, Freund B, Feather D, Abramov R, et al. (2010) Loss of insulin signaling in vascular endothelial cells accelerates atherosclerosis in Apo lipoprotein E null mice. Cell Metab 11: 379-389.

26. Tremolada G, Del Turco C, Lattanzio R, Maestroni S, Maestroni A, et al. (2012) The role of angiogenesis in the development of proliferative diabetic retinopathy: impact of intravitreal anti-VEGF treatment. Exp Diabetes Res 2012:7 28325.

27. Satchell SC (2012) The glomerular endothelium emerges as a key player in diabetic nephropathy. Kidney Int 82: 949-951.

28. Stirban A (2014) Microvascular dysfunction in the context of diabetic neuropathy. Curr Diab Rep 14: 541.

29. Exalto LG, Whitmer RA, Kappele LJ, Biessels GJ (2012) An update on type 2 diabetes, vascular dementia and Alzheimer's disease. Exp Gerontol 47: 858-864.

30. Kanetsuna Y, Takahashi K, Nagata M, Gannon MA, Breyer MD, et al. (2007) Deficiency of endothelial nitric-oxide synthase confers susceptibility to diabetic nephropathy in nephropathy-resistant inbred mice. Am J Pathol 170: 14731484.

31. Zhao HJ, Wang S, Cheng H, Zhang MZ, Takahashi T, et al. (2006) Endothelial nitric oxide synthase deficiency produces accelerated nephropathy in diabetic mice. J Am Soc Nephrol 17: 2664-2669.

32. Huber JD, Bentzien J, Boyer SJ, Burke J, De Lombaert S, et al. (2012) Identification of a potent sodium hydrogen exchanger isoform 1 (NHE1) inhibitor with a suitable profile for chronic dosing and demonstrated cardioprotective effects in a preclinical model of myocardial infarction in the rat. J Med Chem 55: 7114-7140.

33. Sarigianni M, Tsapas A, Mikhailidis DP, Kaloyianni M, Koliakos G, et al. (2010) $\mathrm{Na}+\mathrm{H}+$ exchanger-1: a link with atherogenesis? Expert Opin Investig Drugs 19: 1545-1556.

34. Wang S, Peng Q, Zhang J, Liu L (2008) $\mathrm{Na}+/ \mathrm{H}+$ exchanger is required for hyperglycaemia-induced endothelial dysfunction via calcium-dependent calpain. Cardiovasc Res 80: 255-262.

35. Packer M (2017) Activation and Inhibition of SodiumHydrogen Exchanger Is a Mechanism That Links the Pathophysiology and Treatment of Diabetes Mellitus With That of Heart Failure. Circulation 136: 1548-1559.

36. Katusic ZS, Austin SA (2016) Neurovascular Protective Function of Endothelial Nitric Oxide- Recent Advances. Circ J 80: 1499-1503.

37. Alves-Lopes R, Neves KB, Montezano AC, Harvey A, Carneiro FS, et al. (2016) Internal pudental artery dysfunction in diabetes mellitus is mediated by NOX1-derived ROS-,
Nrf2-, and Rho kinase-dependent mechanisms. Hypertension 68: $1056-1064$.

38. Wu S, Gao X, Yang S, Liu L, Ge B, et al. (2013) Protective effects of cariporide on endothelial dysfunction induced by homocysteine. Pharmacology 92: 303-309.

39. Packer M (2017) Role of the sodium-hydrogen exchanger in mediating the renal effects of drugs commonly used in the treatment of type 2 diabetes. Diabetes Obes Metab 20: 800811.

40. Chang HB, Gao X, Nepomuceno R, Hu S, Sun D (2015) $\mathrm{Na}(+) / \mathrm{H}(+)$ exchanger in the regulation of platelet activation and paradoxical effects of cariporide. Exp Neurol 272: 11-6.

41. Osar Z, Samanci T, Demirel GY, Damci T, Ilkova H (2004) Nicotinamide effects oxidative burst activity of neutrophils in patients with poorly controlled type 2 diabetes mellitus. Exp Diabesity Res 5: 155-162.

42. Hansen SS, Aasum E, Hafstad AD (2017) The role of NADPH oxidases in diabetic cardiomyopathy. Biochim Biophys Acta 1864: 1908-1913.

43. Roy S, Kim D, Hernandez C, Simo R, Roy S (2015) Beneficial effects of fenofibric acid on overexpression of extracellular matrix components, cox-2, and impairment of endothelial permeability associated with diabetic retinopathy. Exp Eye Res 140: 124-129.

44. Othman A, Ahmad S, Megyerdi S, Mussell R, Choksi K, et al. (2013) 12/15-Lipoxygenase- derived lipid metabolites induce retinal endothelial cell barrier dysfunction: Contribution of NADPH oxidase. Ploys One 8: e57254.

45. Zheng Y, He M, Congdon N (2012) The worldwide epidemic of diabetic retinopathy. Indian J Ophthalmol 60: 428-431.

46. Wada J, Makino H (2013) Inflammation and the pathogenesis of diabetic nephropathy Clin Sci (Lond) 124: 139-152.

47. Yang B, Hodgkinson A, Oates PJ, Millward BA, Demaine AG (2008) High glucose induction of DNA-binding activity of the transcription factor NF- $\mathrm{KB}$ in patients with diabetic nephropathy. Biochim Biophys Acta 1782: 295-302.

48. Ha H, Yu MR, Choi YJ, Kitamura M, Lee HB (2002) Role of high glucose-induced nuclear factor- $\mathrm{KB}$ activation in monocyte chemoattractant protein-1 expression by mesangial cells. J Am Soc Nephrol 13: 894-902.

49. Park CW, Kim JH, Lee JH, Kim YS, Ahn HJ, et al. (2000) High glucose-induced intercellular adhesion molecule-1 (ICAM-1) expression through an osmotic effect in rat mesangial cells is PKC-NF- $\kappa$ B-dependent. Diabetologia 43: 1544-1553.

50. Kashihara N, Haruna Y, Kondeti VK, Kanwar YS (2010) Oxidative stress in diabetic nephropathy. Curr Med Chem 17: 4256-4269.

51. Jalal DI, Rivard CJ, Johnson RJ, Maahs DM, McFann K, et al. (2010) Serum uric acid levels predict the development of 
albuminuria over 6 years in patients with type 1 diabetes: findings from the Coronary Artery Calcification in Type 1 Diabetes study. Nephrol Dial Transplant 25: 1865-1869.

52. Ficociello LH, Rosolowsky ET, Niewczas MA, Maselli NJ, Weinberg JM, et al. (2010) High-normal serum uric acid increases risk of early progressive renal function loss in type 1 diabetes: results of a 6-year follow-up. Diabetes Care 33 . 1337-1343.

53. Hovind P, Rossing P, Tarnow L, Johnson RJ, Parving HH (2009) Serum uric acid as a predictor for development of diabetic nephropathy in type 1 diabetes: an inception cohort study. Diabetes 58: 1668-1671.

54. Yan D, Tu Y, Jiang F, Wang J, Zhang R, et al. Uric Acid is independently associated with diabetic kidney disease: a cross-sectional study in a Chinese population. PLoS One 10: e0129797.

55. De Cosmo S, Viazzi F, Pacilli A, Giorda C, Ceriello A, et al. (2015) AMD-Annals Study Group. Serum Uric Acid and Risk of CKD in Type 2 Diabetes. Clin J Am Soc Nephrol 10: 1921-1929.

56. Takae K, Nagata M, Hata J, Mukai N, Hirakawa Y, et al. (2016) Serum Uric Acid as a Risk Factor for Chronic Kidney Disease in a Japanese Community - The Hisayama Study. Circ J 80: 1857-1862.

57. Bartáková V, Kuricová K, Pácal L, Nová Z, Dvořáková V, et al. (2016) Hyperuricemia contributes to the faster progression of diabetic kidney disease in type 2 diabetes mellitus. J Diabetes Complications 30: 1300-1307.

58. Liu P, Chen Y, Wang B, Zhang F, Wang D, et al. (2015) Allopurinol treatment improves renal function in patients with type 2 diabetes and asymptomatic hyperuricemia: 3-year randomized parallel-controlled study. Clin Endocrinol (Oxf) 83: 475-482.

59. Kanji T, Gandhi M, Clase CM, Yang R (2015) Urate lowering therapy to improve renal outcomes in patients with chronic kidney disease: systematic review and meta-analysis. BMC Nephrol 16: 58.

60. Erlandsson Harris H, Andersson U (2004) Mini-review: The nuclear protein HMGB1 as a proinflammatory mediator. Eur J Immunol 34: 1503-1512.

61. Cai W, Duan XM, Liu Y, Yu J, Tang YL, et al. (2017) Uric Acid Induces Endothelial Dysfunction by Activating the HMGB1/RAGE Signaling Pathway. Biomed Res Int 2017: 4391920.

62. Wang J, Qin T, Chen J, Li Y, Wang L, et al. (2014) Hyperuricemia and risk of incident hypertension: a systematic review and meta-analysis of observational studies. PLoS One 9: e114259.

63. Vilsbøll T, Holst JJ (2004) Incretins, insulin secretion and type 2 diabetes mellitus. Diabetologia 47: 357-366.

64. Kalra S (2013) Glucagon-like peptide-1 receptors agonists
(GLP1 RA). J Pak Med Assoc 63: 1312-1315.

65. Ross SA, Ekoé JM (2010) Incretin agents in type 2 diabetes. Can Fam Physician 56: 639-648.

66. Inzucchi SE, Bergenstal RM, Buse JB, Diamant $\mathrm{M}$, Ferrannini E, et al. (2015) Management of hyperglycemia in type 2 diabetes, 2015: a patient-centered approach: update to a position statement of the American Diabetes Association and the European Association for the Study of Diabetes. Diabetes Care 38: 140-149.

67. Sivertsen J, Rosenmeier J, Holst JJ, Vilsbøll T (2012) The effect of glucagon-like peptide 1 on cardiovascular risk. Nat Rev Cardiol 9: 209-222.

68. Drucker DJ (2016) The cardiovascular biology of glucagonlike peptide-1. Cell Metab 24: 15-30.

69. Lepore JJ, Olson E, Demopoulos L, Haws T3, Fang Z4, et al. (2016) Effects of the novel long-acting GLP-1 agonist, albiglutide, on cardiac function, cardiac metabolism, and exercise capacity in patients with chronic heart failure and reduced ejection fraction. JACC Heart Fail 4: 559-566.

70. Margulies KB, Hernandez AF, Redfield MM, Givertz MM, Oliveira GH, et al. (2016) Effects of liraglutide on clinical stability among patients with advanced heart failure and reduced ejection fraction: a randomized clinical trial. JAMA 316: 500-508.

71. Pfeffer MA, Claggett B, Diaz R, Dickstein K, Gerstein HC, et al. (2015) Lixisenatide in patients with type 2 diabetes and acute coronary syndrome. N Engl J Med 373: 2247-2257.

72. Marso SP, Daniels GH, Brown-Frandsen K, Kristensen P, Mann JF, et al. (2016) Liraglutide and cardiovascular outcomes in type 2 diabetes. N Engl J Med 375: 311-322.

73. Marso SP, Bain SC, Consoli A, Eliaschewitz FG, Jódar E, et al. (2016) Semaglutide and cardiovascular outcomes in patients with type 2 diabetes. N Engl J Med 375: 1834-1844.

74. Holman RR, Bethel MA, Mentz RJ, Thompson VP, Lokhnygina Y, et al. (2017) Effects of once-weekly exenatide on cardiovascular outcomes in type 2 diabetes. N Engl J Med 377: 1228-1239.

75. Røder ME (2018) Major adverse cardiovascular event reduction with GLP-1 and SGLT2 agents: evidence and clinical potential. Ther Adv Chronic Dis 9: 33-50.

76. Ferdinand KC, Botros FT, Atisso CM, Sager PT (2016) Cardiovascular safety for once-weekly dulaglutide in type 2 diabetes: a pre-specified meta-analysis of prospectively adjudicated cardiovascular events. Cardiovasc Diabetol 15: 38.

77. Thomas MC (2017) The potential and pitfalls of GLP-1 receptor agonists for renal protection in type 2 diabetes. Diabetes Metab 43: 2S20-2S27.

78. Davies M, Pieber TR, Hartoft-Nielsen ML, Hansen OKH, Jabbour S, et al. (2017) Effect of Oral Semaglutide Compared 
With Placebo and Subcutaneous Semaglutide on Glycemic Control in Patients With Type 2 Diabetes: A Randomized Clinical Trial. JAMA 318: 1460-1470.

79. Aroor A, Zuberek M, Duta C, Meuth A, Sowers JR, et al. (2016) Angiotensin II Stimulation of DPP4 Activity Regulates Megalin in the Proximal Tubules. Int J Mol Sci 17: E780.

80. Girardi AC, Fukuda LE, Rossoni LV, Malnic G, Reboucas NA (2008) Dipeptidyl peptidase IV inhibition downregulates $\mathrm{Na}+-\mathrm{H}+$ exchanger NHE3 in rat renal proximal tubule. Am J Physiol Renal Physiol 294: F414-F422.

81. Muskiet MH, Smits MM, Morsink LM, Diamant M (2014) The gut-renal axis: do incretin-based agents confer renoprotection in diabetes? Nat Rev Nephrol 10: 88-103.

82. Scirica BM, Bhatt DL, Braunwald E, Steg PG, Davidson J, et al. (2013) Saxagliptin and cardiovascular outcomes in patients with type 2 diabetes mellitus. N Engl J Med 369: 1317-1326.

83. Udell JA, Bhatt DL, Braunwald E, Cavender MA, Mosenzon O, et al. (2015) Saxagliptin and cardiovascular outcomes in patients with type 2 diabetes and moderate or severe renal impairment: observations from the SAVOR-TIMI 53 Trial. Diabetes Care 38: 696-705.

84. Eun Lee J, Kim JE, Lee MH, Song HK, Ghee JY, et al. (2016) DA-1229, a dipeptidyl peptidase IV inhibitor, protects against renal injury by preventing podocyte damage in an animal model of progressive renal injury. Lab Invest 96: 547-560.

85. Glorie LL, Verhulst A, Matheeussen V, Baerts L, Magielse J, et al. (2012) DPP4 inhibition improves functional outcome after renal ischemia-reperfusion injury. Am J Physiol Renal Physiol 303: F681-688.

86. Holst JJ, Deacon CF (2005) Glucagon-like peptide-1 mediates the therapeutic actions of DPP-IV inhibitors. Diabetologia 48: 612-615.

87. Nistala R, Savin V (2017) Diabetes, hypertension and chronic kidney disease progression: Role of DPP4. Am J Physiol Renal Physiol 312: F661-F670.

88. Deacon CF (2005) What do we know about the secretion and degradation of incretin hormones? Regul Pept 128: 117-124.

89. De MI, Korom S, Van DJ, Scharpe S (1999) CD26, let it cut or cut it down. Immunol Today 20: 367-375.

90. Klemann C, Wagner L, Stephan M, von Horsten S (2016) Cut to the chase: a review of CD26/dipeptidyl peptidase4's (DPP4) entanglement in the immune system. Clin Exp Immunol 185: 1-21.

91. Lamers D, Famulla S, Wronkowitz N, Hartwig S, Lehr S, et al. (2011) Dipeptidyl peptidase 4 is a novel adipokine potentially linking obesity to the metabolic syndrome. Diabetes 60: 1917-1925.

92. Chowdhury HH, Velebit J, Radić N, Frančič V, Kreft M, et al. (2016) Hypoxia Alters the Expression of Dipeptidyl Peptidase 4 and Induces Developmental Remodeling of Human Preadipocytes. J Diabetes Res 2016: 7481470.

93. Röhrborn D, Eckel J (2014) Sell H Shedding of dipeptidyl peptidase 4 is mediated by metalloproteases and up-regulated by hypoxia in human adipocytes and smooth muscle cells. FEBS Lett. 588: 3870-3877.

94. Girardi AC, Degray BC, Nagy T, Biemesderfer D, Aronson PS (2001) Association of $\mathrm{Na}(+)-\mathrm{H}(+)$ exchanger isoform NHE3 and dipeptidyl peptidase IV in the renal proximal tubule. J Biol Chem 276: 46671-46677.

95. Girardi AC, Fukuda LE, Rossoni LV, Malnic G, Reboucas NA (2008) Dipeptidyl peptidase IV inhibition downregulates $\mathrm{Na}+-\mathrm{H}+$ exchanger NHE3 in rat renal proximal tubule Am J Physiol Renal Physiol 294: F414-F422.

96. Aroor A, Zuberek M, Duta C, Meuth A, Sowers JR, et al. (2016) Angiotensin II Stimulation of DPP4 Activity Regulates Megalin in the Proximal Tubules. Int J Mol Sci 17: E780.

97. Gekle M (2005) Renal tubule albumin transport. Annu Rev Physiol 67: 573-594.

98. Dekan G, Miettinen A, Schnabel E, Farquhar MG (1990) Binding of monoclonal antibodies to glomerular endothelium, slit membranes, and epithelium after in vivo injection. Localization of antigens and bound IgGs by immunoelectron microscopy. Am J Pathol 137: 913-927.

99. Alter ML, Ott IM, von WK, Tsuprykov O, Sharkovska Y, et al. (2012) DPP-4 inhibition on top of angiotensin receptor blockade offers a new therapeutic approach for diabetic nephropathy. Kidney Blood Press Res 36: 119-351.

100. Mosenzon O, Leibowitz G, Bhatt DL, Cahn A, Hirshberg B, et al. (2017) Effect of Saxagliptin on renal outcomes in the SAVOR-TIMI 53 Trial. Diabetes Care, 2017; 40: 69-76.

101. Cornel JH, Bakris GL, Stevens SR, Alvarsson M, Bax WA, et al. (2016) Effect of sitagliptin on kidney function and respective cardiovascular outcomes in type 2 diabetes: Outcomes from TECOS. Diabetes Care 39: 2304-2310.

102. White WB, Cannon CP, Heller SR, Nissen SE, Bergenstal RM, et al. (2013) Alogliptin after acute coronary syndrome in patients with type 2 diabetes. NEngl J Med 369: 1327-1335.

103. Groop PH, Cooper ME, Perkovic V, Hocher B, Kanasaki K, et al. (2017) Linagliptin and its effects on hyperglycaemia and albuminuria in patients with type 2 diabetes and renal dysfunction: The randomized MARLINA-T2D trial. Diabetes Obes Metab 19: 1610-1619.

104. Groop PH, Cooper ME, Perkovic V, Emser A, Woerle HJ, et al. (2013) Linagliptin lowers albuminuria on top of recommended standard treatment in patients with type 2 diabetes and renal dysfunction. Diabetes Care 36: 3460-3468.

105. Kanasaki K, Shi S, Kanasaki M, He J, Nagai T, et al. (2014) Linagliptin-mediated DPP-4 inhibition ameliorates kidney 
fibrosis in streptozotocin-induced diabetic mice by inhibiting endothelial-to-mesenchymal transition in a therapeutic regimen. Diabetes 63: 2120-2131.

106. Shi S, Srivastava SP, Kanasaki M, He J, Kitada M, et al. (2015) Interactions of DPP-4 and integrin $\beta 1$ influences endothelial-to-mesenchymal transition. Kidney Int 88: 479489.

107. Lee CS, Kim YG, Cho HJ, Park J, Jeong H, et al. (2016) Dipeptidyl Peptidase-4 Inhibitor Increases Vascular Leakage in Retina through VE-cadherin Phosphorylation. Sci Rep 6: 29393.

108. Chung YR, Park SW, Kim JW, Kim JH (2016) Protective Effects of Dipeptidyl Peptidase-4 Inhibitors On Progression Of Diabetic Retinopathy In Patients With Type 2 Diabetes. Retina 36: 2357-2363.

Copyright: $\mathbb{C} 2019$ Usama AA Sharaf El Din. This is an open-access article distributed under the terms of the Creative Commons Attribution License, which permits unrestricted use, distribution, and reproduction in any medium, provided the original author and source are credited. 